\title{
Biotin-streptavidin-guided two-step pretargeting approach using PLGA for molecular ultrasound imaging and chemotherapy for ovarian cancer
}

\author{
Hang Zhou ${ }^{1}$, Jing Fu ${ }^{1}$, Qihuan Fu ${ }^{1}$, Yujie Feng ${ }^{1}$, Ruixia Hong ${ }^{1}$, Pan $\mathbf{L i}^{2}$, Zhigang Wang ${ }^{2}$, Xiaoling Huang ${ }^{\text {Corresp., }}{ }^{3}$, \\ Fang Li ${ }^{\text {Corresp. } 1}$ \\ ${ }^{1}$ Ultrasound Medicine Department, ,Chongqing Key Laboratory of Translational Research for Cancer Metastasis and Individualized Treatment, Chongqing \\ University Cancer Hospital, Chongqing, Shapingba District, China \\ 2 Ultrasound Department, Chongqing Key Laboratory of Ultrasound Molecular Imaging, the Second Affiliated Hospital of Chongqing Medical University, \\ Chongqing, Yuzhong District, China \\ 3 Department of ultrasound, the First Hospital Affiliated Chongqing Medical University, Chongqing, Chongqing, Yuzhong District, China \\ Corresponding Authors: Xiaoling Huang, Fang Li \\ Email address: huangxiaoling_4@163.com, 1768308607@qq.com
}

Background Ovarian cancer seriously threatens the lives and health of women, and early diagnosis and treatment are still challenging. Pre-targeting is a promising strategy to improve the treatment efficacy of ovarian cancer and the results of ultrasound imaging.Purpose To explore the effects of a pre-targeting strategy using streptavidin (SA) and paclitaxel (PTX)-loaded phase-shifting poly lactic-co-glycolic acid (PLGA) nanoparticles with perfluoro-n-pentane (PTX-PLGA-SA/PFPs) on the treatment and ultrasound imaging of ovarian cancer.Methods PTX-PLGA/PFPs were prepared with a single emulsion (O/W) solvent evaporation method and SA was attached using carbodiimide. The encapsulation efficiency of PTX and the release characteristics were assessed with high performance liquid chromatography. The phase-change characteristics of the PTX-PLGA-SA/PFPs were investigated. The anti-carcinoembryonic antigen (CEA) antibody (Ab) was covalently attached to PTX-PLGA/PFPs via carbodiimide to create PTX-PLGA-Ab/PFPs. The targeting efficiency of the nanoparticles and the viability of ovarian cancer SKOV3 cells were evaluated in each group using a microscope, flow cytometry, and cell counting kit 8 assays. Results THE PTX-PLGA-SA/PFPs were spheres with a size of $383.0 \pm 75.59 \mathrm{~nm}$. The encapsulation efficiency and loading capability of the nanoparticles for PTX were $71.56 \pm 6.51 \%$ and $6.57 \pm 0.61 \%$, respectively. PTX was burst-released up to $70 \%$ in $2-3 \mathrm{~d}$. When irradiated at $7.5 \mathrm{~W}$ for $3 \mathrm{~min}$, the PTX-PLGA-SA/PFPs visibly enhanced the ultrasonography images $(\mathrm{P}<0.05)$. At temperatures of $45^{\circ} \mathrm{C}$ and $60^{\circ} \mathrm{C}$, the nanoparticles phase-shifted into micro-bubbles and the sizes increased. The binding efficiencies of SA and $\mathrm{Ab}$ to the PTX-PLGA/PFPs were $97.16 \pm 1.20 \%$ and $92.74 \pm 5.75 \%$, respectively. Pretargeting resulted in a high binding efficacy and killing effect on SKOV3 cells $(P<0.05)$. 
Conclusions The two-step pre-targeting process can significantly enhance the targeting ability of PTX-loaded PLGA nanoparticles for ovarian cancer cells and substantially improve the therapeutic efficacy. This technique provides a new method for ultrasonic imaging and precise chemotherapy for ovarian cancer. 
1 Biotin-streptavidin-guided two-step pretargeting approach using PLGA for molecular 2 ultrasound imaging and chemotherapy for ovarian cancer

3 Running titlePre-targeting for ultrasound and therapy

4 Hang Zhou ${ }^{1}$, Jing $\mathrm{Fu}^{1}$, Qihuan $\mathrm{Fu}^{1}$, Yujie Feng ${ }^{1}$, Ruixia Hong ${ }^{1}$, Pan $\mathrm{Li}^{2}$, Zhigang Wang ${ }^{2}$, Xiaoling

$5 \quad$ Huang ${ }^{3 *}$, Fang $\mathrm{Li}^{1 *}$

$6 \quad{ }^{1}$ Chongqing Key Laboratory of Translational Research for Cancer Metastasis and Individualized

7 Treatment, Chongqing University Cancer Hospital, Chongqing, China.

$8{ }^{2}$ Chongqing Key Laboratory of Ultrasound Molecular Imaging, the Second Affiliated Hospital of

9 Chongqing Medical University, Chongqing, China.

$10{ }^{3}$ Department of ultrasound, the First Hospital Affiliated Chongqing Medical University,

11 Chongqing, China.)

13 Corresponding Author 1:

14 Xiaoling Huang

15 No. 1 Youyi Road, Yuzhong District,Chongqing 400010, P.R. China

16 Email address: huangxiaoling_4@163.com

17 Corresponding Author 2:

18 Fang Li (Xiaoling Huang and Fang Li contributed equally)

19 No. 181 Hanyu Road, Shapingba District,Chongqing 400030, P.R. China

20 Email address: 1768308607@qq.com

22 Xiaoling Huang and Fang Li contributed equally. 


\section{Abstract}

Background Ovarian cancer seriously threatens the lives and health of women, and early diagnosis and treatment are still challenging. Pre-targeting is a promising strategy to improve the treatment efficacy of ovarian cancer and the results of ultrasound imaging.

Purpose The aim of this study was to construct a two-step pre-targeting strategy using streptavidin (SA) and paclitaxel (PTX)-loaded phase-shifting poly lactic-co-glycolic acid (PLGA) nanoparticles with perfluoro-n-pentane (PTX-PLGA-SA/PFPs). The effects of the pre-targeting strategy on the treatment and ultrasound imaging of ovarian cancer were then explored.

Methods PTX-PLGA/PFPs were prepared with a single emulsion $(\mathrm{O} / \mathrm{W})$ solvent evaporation method and SA was attached using carbodiimide. The encapsulation efficiency of PTX and the release characteristics were assessed with high performance liquid chromatography. The phasechange characteristics of the PTX-PLGA-SA/PFPs were investigated. The anti-carcinoembryonic antigen (CEA) antibody (Ab) was covalently attached to PTX-PLGA/PFPs via carbodiimide to create PTX-PLGA-Ab/PFPs. The targeting efficiency of the nanoparticles and the viability of ovarian cancer SKOV3 cells were evaluated in each group using a microscope, flow cytometry, and cell counting kit 8 assays.

Results THE PTX-PLGA-SA/PFPs were spheres with a size of $383.0 \pm 75.59 \mathrm{~nm}$. The encapsulation efficiency and loading capability of the nanoparticles for PTX were $71.56 \pm 6.51 \%$ and $6.57 \pm 0.61 \%$, respectively. PTX was burst-released up to $70 \%$ in $2-3 \mathrm{~d}$. When irradiated at 7.5 temperatures of $45^{\circ} \mathrm{C}$ and $60^{\circ} \mathrm{C}$, the nanoparticles phase-shifted into micro-bubbles and the sizes increased. The binding efficiencies of SA and Ab to the PTX-PLGA/PFPs were $97.16 \pm 1.20 \%$ and $92.74 \pm 5.75 \%$, respectively. Pre-targeting resulted in a high binding efficacy and killing effect on SKOV3 cells $(\mathrm{P}<0.05)$.

Conclusions The two-step pre-targeting process can significantly enhance the targeting ability of PTX-loaded PLGA nanoparticles for ovarian cancer cells and substantially improve the therapeutic efficacy. This technique provides a new method for ultrasonic imaging and precise chemotherapy 
51 for ovarian cancer.

52

53 Key words: Paclitaxel, Pre-targeting technology, Molecular imaging; PLGA, Liquid-gas Phase54 shift

55

56 


\section{Introduction}

Ovarian cancer ranks third in morbidity among female reproductive system tumors but first in fatality $^{1,2}$. Although death in patients with ovarian cancer is associated with a variety of factors, there is no denying that early diagnosis and treatment are the keys to reducing mortality. At present, the treatment for ovarian cancer mainly consists of surgery and chemotherapy but conventional chemotherapy drugs have no tumor distribution specificity 3,4 . While attacking tumor cells, chemotherapy drugs can also kill normal cells, resulting in obvious toxic side effects. Consequently, methods for the accurate treatment of tumors are currently a research focus.

Targeted precision treatment for malignant tumors is very promising. Many researchers have employed effector molecules for imaging or treatment with ligands that directly bind to the receptors on tumor tissues; that is, direct targeting, to achieve targeted treatment of tumors ${ }^{5-7}$. Traditional tumor targeting methods are mostly focused on direct targeting. Although the techniques are convenient to use, they are easily affected by the microenvironment in vivo. In addition, direct targeting methods may face problems during the covalent binding of ligands to effector molecules because the functional groups may be prevented from binding to the receptors, resulting in reduced efficacy due to competition ${ }^{8-10}$. Therefore, improving the efficiency of tumor targeting is a challenge.

The concept of pre-targeting originates from radionuclide imaging research and has attracted great attention for tumor treatment and imaging ${ }^{11-13}$. Ding et al reported a pre-targeting strategy using technetium $^{99 \mathrm{~m}}$-labeled dibenzocyclooctyne derivatives to achieve the imaging of glucose metabolism with a high tumor/blood ratio and tumor/muscle ratio ${ }^{14}$. Zhang et al used a two-step biotin-avidin pre-targeting strategy involving poly lactic-co-glycolic acid (PLGA) nanoparticles encapsulating perfluoro-n-pentane (PFP) for high intensity focused ultrasound (HIFU) ablation ${ }^{15}$. However, this technology is rarely used in ultrasound molecular imaging and synergistic treatment research.

PLGA nanoparticles can pass through the endothelial space of the tumor neovascularization system and have become a hot research topic in recent years. Due to the controllability, low toxicity and side effects, high biodegradability, and good biocompatibility, PLGA has been approved by the US Food and Drug Administration (FDA) as a drug delivery vehicle and has been used in 
various studies on tumor treatment and ultrasound imaging ${ }^{16-18}$. Carcinoembryonic antigen (CEA), a broad-spectrum tumor marker, is highly expressed in ovarian cancer, colon cancer, breast cancer, lung cancer, and other malignant tumors, and has been employed as a receptor that targets tumors ${ }^{19}$. Paclitaxel (PTX) is a non-water-soluble broad-spectrum chemotherapeutic drug that needs to be dissolved in organic solvents such as absolute ethanol, which can cause allergic reactions. Therefore, it is necessary to find a method of administration that can effectively reduce or even avoid unnecessary side effects.

The objective of this study was to develop a two-step pre-targeting strategy using streptavidin (SA) and paclitaxel (PTX)-loaded phase-shifting poly lactic-co-glycolic acid (PLGA) nanoparticles with perfluoro-n-pentane (PTX-PLGA-SA/PFPs) and to explore the effects on the treatment and ultrasound imaging of ovarian cancer. Afterward, SA and PTX-loaded ultrasound molecular contrast agents with PFP (PTX-PLGA-SA/PFPs) were added to SKOV3 cells. Due to the high affinity of biotin and SA, low reaction requirements, and multi-level amplification, the PTXPLGA-SA/PFPs could substantially adhere to SKOV3 cells, thereby facilitating targeted ultrasound molecular imaging and chemotherapy for tumors.

\section{Materials and Methods}

\section{Materials}

PLGA-COOH (molecular weight 12,000) was obtained from Daigang Biological Engineering Co., LTD (Jinan, Shandong, China). PTX was purchased from Haoxuan Biological Co., LTD (Xi'an, Shanxi, China). Polyvinyl alcohol (PVA), 1-ethyl-3-(3-dimethylamino) propyl carbodiimide, hydrochloride (EDC), N-hydroxysuccinimide (NHS), Dimethyl sulfoxide (DMSO), 2-(Nmorpholino) ethanesulfonic acid (MES) buffer, and fluorescein isothiocyanate (FITC)-labeled goat anti-rabbit IgG were purchased from Sigma (St. Louis, MO, USA). SA, phycoerythrin (PE)labeled SA (PE-SA), anti-CEA antibody biotinylated anti-CEA antibody (Bio-Ab), the fluorescent dye 4',6-diamidino-2-phenylindole (DAPI), and the fluorescent dye DiI were obtained from Boorson Biotechnology Co., LTD (Beijing, China). Phosphate-buffered saline (PBS), 4\% paraformaldehyde, and cell counting kit 8 (CCK-8) kits were purchased from Baodu Biological Engineering Co., LTD (Wuhan, Hubei, China). PFP was obtained from Strem Chemicals (USA). 
115 Dichloromethane (CH2CL2) was purchased from Beibei Chemical Reagent Company

116 (Chongqing, Sichuan, China). Sodium azide was obtained from Huanyu Biotechnology Co., LTD

117 (Beijing, China). Fetal bovine serum (FBS), RPMI-1640 medium, and 0.25\% Trypsin were

118 purchased from Hyclone Biochemical Products Co., LTD (Wuhan, Hubei, China).

119

120

121

122

123

124

125

126

127

128

129

130

131

132

133

134

135

136

137

138

139

140

141

\section{Preparation of PTX-loaded PLGA nanoparticles and pure PLGA nanoparticles}

PTX-loaded phase-change PLGA nanoparticles (PTX-PLGA/PFPs) were prepared via emulsification $(\mathrm{O} / \mathrm{W})$. Briefly, $50 \mathrm{mg}$ and $5 \mathrm{mg}$ of PTX were dissolved in $2 \mathrm{~mL}$ of dichloromethane and placed in an ice bath. Afterward, PFP and 5\% PVA aqueous solution was added and the mixture was sonicated at $100 \mathrm{~W}$ for $6 \mathrm{~min}$, followed by the addition of $20 \mathrm{~mL}$ of $2 \%(\mathrm{v} / \mathrm{v})$ isopropanol aqueous solution. The reaction was stirred with a magnetic stirrer for $2-3 \mathrm{~h}$ to volatilize the dichloromethane, centrifugated and washed with water several times to obtain PTXPLGA/PFPs, then stored at $4^{\circ} \mathrm{C}$. Pure nanoparticles without PTX (PLGA/PFPs) were prepared using the same protocol.

\section{Preparation of PTX-PLGA-SA/PFPs and PTX-PLGA-Ab/PFPs}

The prepared PTX-PLGA/PFP nanoparticles were suspended in MES buffer (0.1 mol/L, pH 5.5). EDC and NHS were added and the mixture was incubated in a shaker for $45 \mathrm{~min}$. The reaction was centrifuged and washed three times with water to remove EDC and NHS followed by resuspension in MES buffer (0.1 mol/L, pH 8.0). PTX-PLGA/PFP and SA were mixed at a mass ratio of 1:1. Ab was added to PTX-PLGA/PFP (PTX-PLGA/PFP : Ab=20:1) and the mixture was stirred for $2 \mathrm{~h}$ at $4^{\circ} \mathrm{C}$, followed by washing with water several times to obtain PTX-PLGA-SA/PFPs and PTX-PLGA-Ab/PFPs. For fluorescence observation, PE-SA was used to prepare PTX-PLGASA-PE/PFPs in the same manner in the dark. PTX-PLGA-Ab/PFPs stained with DiI were incubated with FITC-labeled IgG for $1 \mathrm{~h}$ in a shaker at $4^{\circ} \mathrm{C}$ and washed with water. The binding of SA and Ab to the PTX-PLGA/PFPs was confirmed using a confocal laser scanning microscope (CLSM) and flow cytometer. 


\section{Characteristic of nanoparticles}

144 145

146

147

148

149

150

151

152

153

154

155

156

157

158

159

160

161

162

163

164

165

166

167

168

The morphology of PTX-PLGA-SA/PFPs was observed with a transmission electron microscope (TEM, Hitachi, Tokyo, Japan). The particle size and zeta potential of the PTX-PLGA/PFPs, PLGA/PFPs, PTX-PLGA-Ab/PFPs, and PTX-PLGA-SA/PFPs were determined with a Malvern particle size analyzer (Brookhaven Instruments Co., Holtsville, USA). High-performance liquid chromatography (HPLC, Waters E2695, Germany) was used to detect the encapsulation efficiency and drug loading of PTX in the PTX-PLGA/PFPs. The liquid-phase conditions were as follows. The mobile phase was acetonitrile water at a ratio of 45:55, the detection wavelength was $227 \mathrm{~nm}$, and the detector was the UV2489 C18 Xbridge $(150 \times 4.6 \mathrm{~mm}, 3.5 \mu \mathrm{m})$. The volume of the organic phase was $2 \mathrm{~mL}$, the temperature was $4{ }^{\circ} \mathrm{C}$, and the stirring speed was $800 \mathrm{rpm}$. The power capacity of the sonicator was $100 \mathrm{~W}$ in pulse mode, which was applied for $5 \mathrm{~s}$ every $5 \mathrm{~s}$, with a total sonicating time of $6 \mathrm{~min}$. PTX was dissolved in methanol to prepare a standard solution at concentrations of $12.5,25,50,75$, and $100 \mu \mathrm{g} / \mathrm{mL}$ and used to create a standard curve. The PTXPLGA/PFPs were demulsified using methyl chloride and the PTX content was determined using HPLC. PTX encapsulation rate $(\%)=($ Detection amount $/$ total dose $) \times 100 \%$. Drug loading capacity $(\%)=($ Detection amount/total mass of nanoparticles $) \times 100 \%$.

\section{In vitro release characteristics of PTX-loaded nanoparticles}

Two mL of PTX-PLGA/PFPs nanoparticles (resuspended in PBS) was added to a dialysis bag and placed in $150 \mathrm{~mL}$ of medium containing 30\% ethanol, $0.01 \%$ TWEEN-80, and $0.02 \%$ azide sodium. Subsequently, the mixture was placed on a shaker at $37^{\circ} \mathrm{C}$ and shaken at $150 \mathrm{rpm}$. Then, $1 \mathrm{~mL}$ of the solution was removed at $2,4,8,12,24,48,72,96,120,144$, and $168 \mathrm{~h}$, respectively, and $1 \mathrm{~mL}$ of fresh solution was added. The PTX content was determined using HPLC. ultrasound imaging in vitro and thermally induced phase change 
PTX-PLGA-SA/PFPs nanoparticles were placed in the wells of a gel model and irradiated with LIFU at different power levels $(3.3,4.4,5.5,6.5,7.5,8.5 \mathrm{~W})$ for $3 \mathrm{~min}$ before being observed in normal mode (B-mode) and contrast-enhanced ultrasound (CEUS). The average echo intensities of the ultrasound images before and after the phase change were compared using the DFY image analysis system. After determining the optimal power, LIFU irradiation was performed at $0.1,0.5$, $1.0,2.0$, and $5.0 \mathrm{mg} / \mathrm{mL}$ of the nanoparticle concentration (based on the quality of PLGA). In addition, the heating plate method was used to observe the thermally induced phase transition process for the nanoparticles under a microscope.

\section{Cell culture}

Human ovarian cancer SKOV3 cells were cultured with RPMI 1640 medium containing 10\% FBS and $1 \%$ penicillin-streptomycin with $5 \% \mathrm{CO}_{2}$ at $37^{\circ} \mathrm{C}$. The cells were cultured in a constant temperature incubator (the incubation processes described later were performed under this condition). The cells were digested with $0.25 \%$ trypsin and centrifuged at $1000 \mathrm{rpm}$ for $5 \mathrm{~min}$.

\section{Cellular immunofluorescence experiment}

SKOV3 cells in the logarithmic growth phase were cultured in a disposable $15 \mathrm{~mm}$ glass-bottom dish, incubated with $\mathrm{Ab}$ for $2 \mathrm{~h}$, and washed with PBS, followed by adding FITC-labeled IgG (secondary antibody) for $1 \mathrm{~h}$. The cells were fixed with 4\% paraformaldehyde for 15-20 min, washed with PBS three to five times, incubated with DAPI for 5-8 minutes, washed with PBS three to five times, and observed under a CLSM.

\section{Cytotoxicity of PTX-PLGA/PFPs}

SKOV3 cells were seeded in a 96-well plate at a concentration of $10^{4}$ cells/well and incubated with PTX-PLGA/PFPs at concentrations of 1.0, 2.0, 3.0, 4.0, and $5.0 \mathrm{mg} / \mathrm{mL}$ (calculated based on PLGA). Cells without any treatment were used as the negative controls and each group had five replicates. After $24 \mathrm{~h}$ or $48 \mathrm{~h}$, the cells were washed with PBS three to five times, and the cell 
196

197

198

199

200

201

202

203

204

205

206

207

208

209

210

211

212

213

214

215

216

217

218

219

220

221

222

223

survival rate was assessed with the CCK-8 assay according to the instructions for the kit. Cell survival rate $(\mathrm{v} \%)=($ optical density $(\mathrm{OD})$ of experimental group - OD of zero adjustment group) / (OD of negative group - OD of zero adjustment group) $* 100 \%$.

\section{In vitro targeting experiment}

Cells were cultured in a disposable dish and divided into a drug-loaded pre-targeting group, drugloaded direct-targeting group, drug-loaded non-targeting group, and antibody-blocking group. In the pre-targeting group, the cells were first incubated with $200 \mu \mathrm{L}$ of Bio-Ab at a concentration of $100 \mu \mathrm{g} / \mathrm{mL}$ for $2 \mathrm{~h}$, washed with PBS three to five times, and then incubated with $1 \mathrm{~mL}$ of DiIlabeled PTX-PLGA-SA/PFPs. In the drug-loaded direct-targeting group, nanoparticles and the same amount of DiI-PTX-PLGA-Ab/PFPs were added. In the drug-loaded non-targeting group, DiI-PTX-PLGA/PFPs were added. The procedure for the antibody blocking group was the same as that for the drug-loaded pre-targeting group, but Bio-Ab was replaced with the same amount of Ab. All cells were further incubated for $45 \mathrm{~min}$, washed with PBS several times, fixed with 4\% paraformaldehyde for $20 \mathrm{~min}$, washed with PBS three to five times, stained with DAPI, washed with PBS, and observed under a CLSM. In addition, the cells were cultured in a six-well plate with approximately $10^{6}$ cells per well. The blank control group consisted of cells without any treatment. After adding the corresponding nanoparticles or reagents to each group, the cells were cultured for 45 minutes and washed with PBS. The cells were digested with $0.25 \%$ trypsin, centrifuged at 800 rpm, resuspended in $1 \mathrm{~mL}$ of PBS, and visualized with flow cytometry.

\section{In vitro toxicity to SKOV3 cells}

SKOV3 cells were seeded in 96-well plates at a concentration of $10^{4}$ cells/well and divided into a drug-loaded pre-targeting group, drug-loaded direct-targeting group, drug-loaded non-targeted group, antibody-blocking group, nanoparticle only group, free drug group, and blank control group. Each group had five replicates. In the drug-loaded pre-targeting group, the cells were first incubated with $25 \mathrm{uL}$ of Bio-Ab at a concentration of $100 \mathrm{ug} / \mathrm{mL}$. After incubating for $2 \mathrm{~h}$, the cells were washed with PBS three to five times and incubated with $100 \mathrm{uL}$ PTX-PLGA-SA/PFPs. 
224 The procedure for the antibody blocking group was the same as that for the drug-loaded pre225 targeting group, but Bio-Ab was replaced with the same amount of Ab. In the drug-loaded direct 226 targeting group, PTX-PLGA-Ab/PFPs were added. In the drug-loaded non-targeting group, PTX227 PLGA/PFPs were added. In the nanoparticle only group, the same amount of PLGA/PFPs was 228 added to each well. In the free drug group, an equal amount of fresh 1640 medium containing PTX $229(10 \mathrm{ug} / \mathrm{mL})$ was added to each well. In the blank control group, $100 \mathrm{uL}$ of fresh $1640 \mathrm{medium}$ was 230 added to each well. After adding the corresponding nanoparticles or reagents, the cells were 231 cultured for $45 \mathrm{~min}$, washed with PBS three to five times, and $100 \mathrm{uL}$ of fresh RMPI 1640 medium 232 was added. After $24 \mathrm{~h}$ or $48 \mathrm{~h}$ of incubation, the CCK-8 assay was used to determine the cell 233 viability.

\section{Statistical analysis}

236

237

238

239

240

241

242

243

244

245

246

247

248

249

250

All statistical analysis was performed using SPSS 19.0 statistical analysis software. The independent sample t test was used for comparison between two groups and single factor analysis of variance was used to compare the means of multiple groups. The least significant difference (LSD) test was used for pairwise comparisons between multiple groups and $\mathrm{P}<0.05$ was considered as statistically significant.

\section{Result}

\section{Preparation and characterization of PLGA}

The prepared PTX-PLGA-SA/PFPs were spheres with a uniform size (Figure 1A-D). The diameters of the PTX-PLGA/PFPs, PLGA/PFPs, PTX-PLGA-Ab/PFPs, and PTX-PLGASA/PFPs were $333.0 \pm 52.27,364.9 \pm 106.9,397.7 \pm 99.96$, and $383.0 \pm 75.59 \mathrm{~nm}$, respectively (Figure 1E). The zeta potentials of the PTX-PLGA/PFPs, PLGA/PFPs, PTX-PLGA-Ab/PFPs, and PTXPLGA-SA/PFPs were $-15.70 \pm 3.07,-32.7 \pm 6.81,-0.93 \pm 3.43$, and $-5.66 \pm 3.46 \mathrm{mV}$, respectively. The zeta potentials of the PTX-PLGA/PFPs without SA or Ab and PLGA/PFPs without drugs were significantly higher than those for the other nanoparticles $(\mathrm{P}<0.05)$. 
252

253

254

255

256

257

258

259

260

261

262

263

264

265

266

267

268

269

270

271

272

273

274

275

276

277

\section{PTX load and release}

The PTX encapsulation rate and loading capacity were $71.56 \pm 6.51 \%$ and $6.57 \pm 0.61 \%$, respectively (Figure 1F). PTX was released relatively quickly in the first $48 \mathrm{~h}$ and the drug release rate exceeded $70 \%$ after nearly 50 hours. After this period, the drug release rate became significantly slower, showing obvious burst-release characteristics with a high drug release rate (Figure 1G).

\section{Connection between SA and Ab and PTX-PLGA/PFPs}

Orange fluorescence was observed when the PE-SA attached to the fluorescent PTX-PLGA/PFPs, indicating that SA successfully attached to the nanoparticles. The fluorescence was yellow when FITC-labeled IgG attached to the DiI-labeled PTX-PLGA/PFPs, demonstrating the Ab successfully attached to the PTX-PLGA/PFPs (Figure 1H). The mean connection rate for SA to PTX-PLGA/PFPs measured with flow cytometry was $97.16 \pm 1.20 \%$, and that of Ab was $92.74 \pm 5.75 \%$ (Figure 2A-D).

\section{In vitro LIFU-induced PTX-PLGA/PFPs phase transition for ultrasound imaging}

After 3 min of LIFU with ultrasound irradiation with a power of 3.3, 4.4, 5.5, 6.5, 7.5, and 8.5 W, each group exhibited significantly higher echo intensity in B-mode, and the echo intensity was highest at $6.5 \mathrm{~W}$ and $7.5 \mathrm{~W}(\mathrm{P}<0.05)$. However, the difference between the echo intensity at 6.5 $\mathrm{W}$ and $7.5 \mathrm{~W}$ was not statistically significant $(\mathrm{P}>0.05)$. In CE-mode, the echo intensity increased with the increase of ultrasound power, reaching the highest value at $7.5 \mathrm{~W}(\mathrm{P}<0.01)$ but decreased at $8.5 \mathrm{~W}$ (Figure $3 \mathrm{~A}-\mathrm{C})$. The echo intensity for different concentrations of nanoparticles $(0.1,0.5$, $1.0,2.0,5.0 \mathrm{mg} / \mathrm{mL}$ ) irradiated with ultrasound at $7.5 \mathrm{~W}$ for $3 \mathrm{~min}$ was investigated. The results showed that the phase change caused by LIFU had a certain relationship with the concentration. When the concentration exceeded $1.0 \mathrm{mg} / \mathrm{mL}$, more obvious images could be obtained in B-mode, and when the concentration exceeded $2.0 \mathrm{mg} / \mathrm{mL}$, the resolution of the CE-mode was significantly 
278 enhanced (Figure 3D, E). There was no significant difference in the echo intensity between 2.0

279 and $5.0 \mathrm{mg} / \mathrm{mL}$ in the B-mode and CE-mode $(\mathrm{P}>0.05)$.

\section{Heat-induced PTX-PLGA-SA/PFPs phase transition}

282

The particle size of PTX-PLGA-SA/PFPs before heating was at the nanometer level and the distribution was uniform. When the temperature of the heating plate reached $45^{\circ} \mathrm{C}$, some of the nanoparticles began to undergo a phase change and gradually increased in size from nanometers to micrometers. When the temperature of the heating plate reached $60^{\circ} \mathrm{C}$, most of the nanoparticles underwent a phase change, with different particle sizes ranging from a few microns to tens of microns. Some of the microbubbles ruptured after increasing in size to a certain extent (Figure 3F).

\section{Cellular immunofluorescence and viability of cells incubated with PTX-PLGA/PFPs}

The products from the two-step biotin-streptavidin pre-targeting technology were combined with PTX-loaded phase-change PLGA multifunctional nanoparticles to treat ovarian cancer (Figure 4A). Green fluorescence was observed, indicating that the FITC-labeled IgG adhered to the cell membrane and the SKOV3 cells expressed a large amount of CEA antigens (Figure 4B). CCK-8 assay results showed that the PTX-PLGA/PFPs killed the SKOV3 cells in a concentrationdependent manner. As the concentration of the nanoparticles increased, the survival rate of the SKOV3 cells at $24 \mathrm{~h}$ and $48 \mathrm{~h}$ gradually decreased (Figure 5A)

\section{Cell targeting and killing effect on tumor cells for nanoparticles in vitro}

Stronger red fluorescent could be observed with CLSM, demonstrating that more DiI-labeled nanoparticles adhered to the SKOV3 cells compared with the drug-loaded direct-targeting group and the other groups (Figure 5B). Flow cytometry results showed that the fluorescence intensity of the drug-loaded pre-targeting group was significantly stronger than that for the other groups, and the difference was statistically significant $(\mathrm{P}<0.05)$ (Figure $5 \mathrm{C}-\mathrm{F})$. The fluorescence intensity 
304 of the drug-loaded direct-targeting group was higher than that for the drug-loaded non-targeting $305 \operatorname{group}(\mathrm{P}<0.05)$.

306 The results of the CCK-8 assay showed that when incubated for $24 \mathrm{~h}$, the cell survival rate for the 307 free drug group was the lowest $(\mathrm{P}<0.05)$ and the difference was statistically significant. The cell 308 survival rate for the drug-loaded pre-targeting group was close to $40 \%$, which was lower than that 309 of the drug-loaded direct-targeting group and the other groups except for the free drug group. The 310 difference was statistically significant $(\mathrm{P}<0.05)$. At $48 \mathrm{~h}$, the cell survival rate for the drug-loaded 311 pre-targeting group decreased to about 5\%, which was lower than that for the other groups except 312 the free drug group, and the difference was statistically significant $(\mathrm{P}<0.05)$. At both $24 \mathrm{~h}$ and 48 $313 \mathrm{~h}$, the cell survival rate for the free drug group was the lowest $(\mathrm{P}<0.05)$ (Figure 6).

\section{Discussion}

316 The morbidity and fatality rates of ovarian cancer in women are very high and early diagnosis and treatment for the disease are challenging. In this study, SA and PTX-loaded phase-shifting PLGA nanoparticles (PTX-PLGA-SA/PFPs) were successfully fabricated with high encapsulation efficiency for PTX and conjugating efficiency for SA. With a burst release in vitro, PTX was released from the nanoparticles at a high rate. Phase transition was induced with LIFU and heating, and after phase transformation, the nanoparticles visibly increased the quality of contrast-enhanced ultrasonic imaging. At present, tumor-targeting PLGA nanoparticles loaded with drugs, genes, etc are a research hotspot in ultrasonic molecular imaging and therapy. Loading the chemotherapeutic drug PTX into phase-shifting PLGA nanoparticles and targeting ovarian cancer cells can not only improve ultrasonic molecular imaging for the early diagnosis of ovarian cancer, but can also alleviate the drug delivery limitations caused by hydrophobicity, increase drug effectiveness, and reduce the side effects.

Direct targeting is one of the most commonly used traditional methods for tumor targeting due to the simplicity of operation but is susceptible to the microenvironment in vivo. In addition, using antibodies as an example, the randomness of covalent binding between the ligand and effector might cause the partial competitive inhibition of binding between functional groups and receptors, leading to a reduction in the targeting ability. Therefore, methods to improve the efficiency of 
333 tumor cell targeting have become a crucial problem which scholars are trying to solve. The 334 antibody molecules used as ligands, regardless of their Fab or Fc segments, are compatible with 335 PLGA. However, the connecting positions are random, which may inhibit the binding of the Fab 336 segment to the receptor due to competition, resulting in reduced targeting.

337 Pre-targeting technology can effectively improve the tumor target/non-target (T/NT) ratio. This study was conducted to develop PTX-loaded and phase-shifting PLGA nanoparticles. Using a twostep biotin-SA pre-targeting technology for targeting ovarian cancer cells in vitro, we aimed to explore whether the pre-targeting technique is a feasible strategy for tumor targeting to improve ultrasonic molecular imaging and treatment. The technique provides a new approach for the accurate diagnosis and treatment of ovarian cancer. The two-step biotin-avidin pre-targeting technology has the characteristics of strong binding in vivo and multi-level amplification and has been applied in other imaging fields ${ }^{20}$. The T/NT value using a pre-targeting technique is significantly higher than that of direct targeting methods ${ }^{21-23}$. However, this method is rarely used in ultrasound molecular targeting research. Avidin was replaced with SA, which is a derivative of avidin with a lower isoelectric point, no glycosyl base, lower non-specific binding affinity, and higher biosafety ${ }^{24-26}$. In the current research, cell fluorescence immunoassays showed that a large amount of FITC-labeled secondary antibody adhered to the cell membrane area of SKOV3 cells, confirming that CEA can be expressed in large quantities in the SKOV3 cell membrane.

In this study, PTX-PLGA/PFPs were prepared using the single emulsification solvent evaporation method $(\mathrm{O} / \mathrm{W})$, which has simple operating steps and a high encapsulation rate for fat-soluble drugs. The results showed that the nanoparticles prepared with this method have a high encapsulation rate for PTX, which is consistent with related literature reports ${ }^{27-29}$. SA was covalently bonded to the surface of the nanoparticles; therefore, we anticipate that it will not affect the encapsulation efficiency for PTX. For the in vitro release of PTX, neutral PBS was used as the dialysis environment, and the slow-release medium was configured according to the fat solubility characteristics of PTX. The in vitro release curve for PTX showed that the drug was burst-released and reached peak release within 2 to 3 days. It can be speculated that the release rate for PTX will be faster in vivo due to the metabolism of PLGA and the released amount would be higher. Simple nanoparticles (PLGA/PFPs) without PTX were also prepared as the control for further cytotoxicity experiments. 
363

364

365

366

367

368

369

370

371

372

373

374

375

376

377

378

379

380

381

382

383

384

385

386

387

388

389

390

391

Direct-target PLGA nanoparticles attached to anti-CEA antibodies were prepared and the targeting efficiency was compared between the pre-targeting technique and direct targeting methods. The connection between $\mathrm{SA}$ and $\mathrm{Ab}$ and the nanoparticles was established using the carbodiimide method, which is simple to perform, has high binding efficiency, and forms a stable connection between nanoparticles and ligands. Flow cytometry was used to semi-quantitatively evaluate the connection between SA, Ab, and the PTX-PLGA/PFPs and the results showed that the connection rate for the $\mathrm{Ab}$ group was lower than that for the SA group. This may be because SA was labeled with PE directly, but the immunofluorescence method was indirectly used to analyze the binding of $\mathrm{Ab}$ through FITC-labeled secondary antibodies. In order to reduce the difficulty of LIFUinduced phase change in nanoparticles, we selected PFP, which has a boiling point of $29{ }^{\circ} \mathrm{C}$, as the phase change material. The experimental results showed that the temperature at which the nanoparticles underwent phase change was significantly higher than that of PFP, which is consistent with the results in other studies ${ }^{30}$. We speculated that this may be due to the obstacle presented by the solid shell to the vaporization and diffusion of the inner core and the influence of the Laplace force inside the nanoparticles. However, these factors are also conducive to the stability of the nanoparticles in vivo.

In the in vitro targeting experiment, the pre-targeting technique improved the targeted adhesion ability of the PTX-loaded phase-change PLGA nanoparticles to ovarian cancer SKOV3 cells. In the current study, the target nanoparticles were incubated with the cells for $45 \mathrm{~min}$, which is shorter than the $>2$-h duration used in other studies ${ }^{31-33}$. Shortening the co-incubation time for the cells and nanoparticles may reflect the time line of the connection between biotin and SA.

There are several limitations in this study. First, the optimization of the in vitro LIFU-induced phase transition conditions for the nanoparticles was insufficient and the influence of SA on the PTX drug loading was not studied. Second, the encapsulation efficiency of liquid fluorocarbon (PFP) was not measured and the release profiles were only investigated in vitro. Third, only the cell survival rate was evaluated and the influence of the nanoparticles on the cell cycle was not studied.

In conclusion, PTX-containing phase-change PLGA nanoparticles targeting ovarian cancer 
392 SKOV3 cells were developed using a biotin-SA two-step pre-targeting process. The research 393 results showed that the pre-targeting technology can improve the in vitro targeted adhesion ability 394 of PTX phase-change PLGA ultrasound molecular contrast agents to ovarian cancer SKOV3 cells. 395 Compared with direct targeting, this technique exhibited better targeting and killing of tumor cells. 396 The results provide a good experimental foundation for further research on pre-positioned 397 ultrasound molecular imaging technology in vivo and also provide a new idea for targeted 398 ultrasound molecular imaging and the treatment of ovarian cancer. 
401

402

403

404

405

406

407

408

409

410

411

412

413

414

415

416

417

418

419

420

421

422

423

424

425

426

\section{References}

1. Goncalves, V.; Jayson, G.; Tarrier, N., A longitudinal investigation of psychological morbidity in patients with ovarian cancer. Br J Cancer 2008, 99 (11), 1794-801.

2. Schmid, B. C.; Oehler, M. K., New perspectives in ovarian cancer treatment. Maturitas 2014, 77 (2), 128-36.

3. Banerjee, S.; Kaye, S. B., New strategies in the treatment of ovarian cancer: current clinical perspectives and future potential. Clin Cancer Res 2013, 19 (5), 961-8.

4. Lopez, J.; Banerjee, S.; Kaye, S. B., New developments in the treatment of ovarian cancer-future perspectives. Ann Oncol 2013, 24 Suppl 10, x69-x76.

5. Stachelek, G. C.; Grimm, J.; Moore, J.; Huang, E.; Spoleti, N.; Redmond, K. J.; Lim, M.; Bettegowda, C.; Kleinberg, L., Tumor-Treating Field Arrays Do Not Reduce Target Volume Coverage for Glioblastoma Radiation Therapy. Adv Radiat Oncol 2020, 5 (1), 62-69.

6. Sun, P.; Zhang, S. J.; Maksim, S.; Yao, Y. F.; Liu, H. M.; Du, J., Epigenetic Modification in Macrophages: A Promising Target for Tumor and Inflammation-associated Disease Therapy. Curr Top Med Chem 2019, 19 (15), 1350-1362.

7. Kato, S.; Okamura, R.; Mareboina, M.; Lee, S.; Goodman, A.; Patel, S. P.; Fanta, P. T.; Schwab, R. B.; Vu, P.; Raymond, V. M.; Lanman, R. B.; Sicklick, J. K.; Lippman, S. M.; Kurzrock, R., Revisiting Epidermal Growth Factor Receptor (EGFR) Amplification as a Target for Anti-EGFR Therapy: Analysis of Cell-Free Circulating Tumor DNA in Patients With Advanced Malignancies. JCO Precis Oncol 2019, 3.

8. Jung, K. H.; $\quad$ Song, S. H.; Paik, J. Y.; Koh, B. H.; Choe, Y. S.; Lee, E. J.; Kim, B. T.; Lee, K. H., Direct targeting of tumor cell F(1)F(0) ATP-synthase by radioiodine angiostatin in vitro and in vivo. Cancer Biother Radiopharm 2007, 22 (5), 704-12.

9. Kennel, S. J.; Foote, L. J.; $\quad$ Lankford, P. K.; Johnson, M.; Mitchell, T.; Braslawsky, G. R., Direct binding of radioiodinated monoclonal antibody to tumor cells: significance of antibody purity and affinity for drug targeting or tumor imaging. Hybridoma 1983, 2 (3), 297-310. 
427 10. Koo, K. H.; Kwon, H., MicroRNA miR-4779 suppresses tumor growth by inducing apoptosis 428 and cell cycle arrest through direct targeting of PAK2 and CCND3. Cell Death Dis 2018, 9 (2), 42977.

430 11. Karacay, H.; Sharkey, R. M.; McBride, W. J.; Griffiths, G. L.; Qu, Z.; Chang, K.; 431 Hansen, H. J.; Goldenberg, D. M., Pretargeting for cancer radioimmunotherapy with bispecific 432 antibodies: role of the bispecific antibody's valency for the tumor target antigen. Bioconjug Chem 433 2002, $13(5), 1054-70$.

434 12. Kraeber-Bodere, F.; Rousseau, C.; Bodet-Milin, C.; Frampas, E.; Faivre-Chauvet, A.; 435 Rauscher, A.; Sharkey, R. M.; Goldenberg, D. M.; Chatal, J. F.; Barbet, J., A pretargeting 436 system for tumor PET imaging and radioimmunotherapy. Front Pharmacol 2015, 6, 54.

437 13. Zhu, H.; Jain, R. K.; Baxter, L. T., Tumor pretargeting for radioimmunodetection and 438 radioimmunotherapy. $J$ Nucl Med 1998, 39 (1), 65-76.

439 14. Ding, J.; Su, H.; Wang, F.; Chu, T., A pre-targeting strategy for imaging glucose 440 metabolism using technetium-99m labelled dibenzocyclooctyne derivative. Bioorg Med Chem Lett 441 2019, $29(14), 1791-1798$.

442 15. Zhang, Y.; Yong, L.; Luo, Y.; Ding, X.; Xu, D.; Gao, X.; Yan, S.; Wang, Q.; 443 Luo, J.; Pu, D.; Zou, J., Enhancement of HIFU ablation by sonosensitizer-loading liquid 444 fluorocarbon nanoparticles with pre-targeting in a mouse model. Sci Rep 2019, 9 (1), 6982.

445 16. Kazi, J.; Sen, R.; Ganguly, S.; Jha, T.; Ganguly, S.; Chatterjee Debnath, M., Folate 446 decorated epigallocatechin-3-gallate (EGCG) loaded PLGA nanoparticles; in-vitro and in-vivo 447 targeting efficacy against MDA-MB-231 tumor xenograft. Int J Pharm 2020, 585, 119449.

448 17. Allavena, P.; Palmioli, A.; Avigni, R.; Sironi, M.; La Ferla, B.; Maeda, A., PLGA 449 Based Nanoparticles for the Monocyte-Mediated Anti-Tumor Drug Delivery System. J Biomed $450 \quad$ Nanotechnol 2020, 16 (2), 212-223.

451 18. Alfaifi, M. Y.; Shati, A. A.; Elbehairi, S. E. I.; Fahmy, U. A.; Alhakamy, N. A.; Md, 452 S., Anti-tumor effect of PEG-coated PLGA nanoparticles of febuxostat on A549 non-small cell 453 lung cancer cells. 3 Biotech 2020, 10 (3), 133. 
454 19. Meller, B.; Rave-Franck, M.; Breunig, C.; Schirmer, M.; Baehre, M.; Nadrowitz, R.; 455 Liersch, T.; Meller, J., Novel Carcinoembryonic-Antigen-(CEA)-Specific Pretargeting System to 456 Assess Tumor Cell Viability after Irradiation of Colorectal Cancer Cells. Strahlenther Onkol 2011, $457 \quad 187(2), 120-6$.

20. Qiu, L. H.; Zhang, J. W.; Li, S. P.; Xie, C.; Yao, Z. W.; Feng, X. Y., Molecular imaging of angiogenesis to delineate the tumor margins in glioma rat model with endoglin-targeted paramagnetic liposomes using 3T MRI. J Magn Reson Imaging 2015, 41 (4), 1056-64.

21. Hapuarachchige, S.; Artemov, D., Theranostic Pretargeting Drug Delivery and Imaging Platforms in Cancer Precision Medicine. Front Oncol 2020, 10, 1131.

22. Hapuarachchige, S.; Huang, C. T.; Donnelly, M. C.; Barinka, C.; Lupold, S. E.; Pomper, M. G.; Artemov, D., Cellular Delivery of Bioorthogonal Pretargeting Therapeutics in PSMA-Positive Prostate Cancer. Mol Pharm 2020, 17 (1), 98-108.

23. Imberti, C.; Adumeau, P.; Blower, J. E.; Al Salemee, F.; Baguna Torres, J.; Lewis, J. S.; Zeglis, B. M.; Terry, S. Y. A.; Blower, P. J., Manipulating the In Vivo Behaviour of (68)Ga with Tris(Hydroxypyridinone) Chelators: Pretargeting and Blood Clearance. Int J Mol Sci 2020, 21 (4).

24. Nguyen, T. T.; Sly, K. L.; Conboy, J. C., Comparison of the energetics of avidin, streptavidin, neutrAvidin, and anti-biotin antibody binding to biotinylated lipid bilayer examined by second-harmonic generation. Anal Chem 2012, 84 (1), 201-8.

25. Paganelli, G.; Magnani, P.; Fazio, F., Pretargeting of carcinomas with the avidin-biotin system. Int J Biol Markers 1993, 8 (3), 155-9.

26. Wilbur, D. S.; Hamlin, D. K.; Vessella, R. L.; Stray, J. E.; Buhler, K. R.; Stayton, P. S.; Klumb, L. A.; Pathare, P. M.; Weerawarna, S. A., Antibody fragments in tumor pretargeting. Evaluation of biotinylated Fab' colocalization with recombinant streptavidin and avidin. Bioconjug Chem 1996, 7 (6), 689-702.

27. Sadat Tabatabaei Mirakabad, F.; Nejati-Koshki, K.; Akbarzadeh, A.; Yamchi, M. R.; Milani, M.; Zarghami, N.; Zeighamian, V.; Rahimzadeh, A.; Alimohammadi, S.; 
481 Hanifehpour, Y.; Joo, S. W., PLGA-based nanoparticles as cancer drug delivery systems. Asian 482 Pac J Cancer Prev 2014, 15 (2), 517-35.

483 28. Klose, D.; Siepmann, F.; Elkharraz, K.; Siepmann, J., PLGA-based drug delivery systems: 484 importance of the type of drug and device geometry. Int J Pharm 2008, 354 (1-2), 95-103.

485 29. Semete, B.; Booysen, L.; Lemmer, Y.; Kalombo, L.; Katata, L.; Verschoor, J.; Swai, 486 H. S., In vivo evaluation of the biodistribution and safety of PLGA nanoparticles as drug delivery 487 systems. Nanomedicine 2010, 6 (5), 662-71.

30. Mountford, P. A.; Thomas, A. N.; Borden, M. A., Thermal activation of superheated lipidcoated perfluorocarbon drops. Langmuir 2015, 31 (16), 4627-34.

31. Costanzo, M.; Carton, F.; Marengo, A.; Berlier, G.; Stella, B.; Arpicco, S.; Malatesta, M., Fluorescence and electron microscopy to visualize the intracellular fate of nanoparticles for drug delivery. Eur J Histochem 2016, 60 (2), 2640.

32. Nsanzamahoro, S.; Cheng, W.; Mutuyimana, F. P.; Li, L.; Wang, W.; Ren, C.; Yi, T.; Chen, H.; Chen, X., Target triggered fluorescence "turn-off" of silicon nanoparticles for cobalt detection and cell imaging with high sensitivity and selectivity. Talanta 2020, 210, 120636.

33. Luo, J.; Gong, T.; Ma, L., Chondroitin-modified lipid nanoparticles target the Golgi to 
500

501

502

503

504

505

506

507

508

509

510

511

512

513

514

515

516

\section{Figure Legends}

Figure 1. Light microscope and electron microscope images of PTX-PLGA-SA/PFPs.

(A) light microscope image. (B) inverted fluorescence microscope image of PTX-PLGA-SA/PFPs labeled with DiI showing as red fluorescence. (C) SEM image. (D) TEM image. (E) Size distributions of PTXPLGA/PFPs, PLGA/PFPs, PTX-PLGA-Ab/PFPs, and PTX-PLGA-SA/PFPs. (F) The chromatography results for PTX encapsulated in nanoparticles in HPLC. (G) The release curve for PTX from PTXPLGA/PFPs in vitro. (H) Confocal laser scanning microscope images of PE labeled SA, Dil labeled PTX-PLGA-Ab/PFPs, Nanoparticles without fluorescence, and FITC labeled IgG.

Figure 2. Flow cytometry analysis of the connection efficiency of both SA and Ab bound to PTXPLGA/PFPs.

Flow cytometry analysis of the connection efficiency of both SA and Ab bound to PTXPLGA/PFPs. (A) Control; (B) SA group; (C) Control; (D) Ab group.

\section{Figure 3. Phase shifting of PTX-PLGA-SA/PFPs.}

(A) After exposure to LIFU at different power levels, the B-mode and contrast ultrasonic images of nanoparticles at 3.3, 4.4, 5.5, 6.5, 7.5, and $8.5 \mathrm{~W}$ are shown. After irradiation with LIFU at different power levels, the average echo intensities of the nanoparticles are shown. (B) B-mode; (C) CEUS mode. “*” indicates there is a significant difference compared with the $0.1 \mathrm{mg} / \mathrm{ml}$ group $(\mathrm{P}<0.05)$; “\#" indicates there is no significant difference between the two groups $(\mathrm{P}>0.05)$. "\&" indicates there is a significant difference compared with the rest of the groups $(\mathrm{P}<0.05)$. The average ultrasonic echo intensity $(\mathrm{dB})$ of the nanoparticles after phase transformation induced by LIFU at different concentrations. (D) B-mode; (E) CEUS. “*” indicates there is a significant difference compared with the $0.1 \mathrm{mg} / \mathrm{ml}$ group $(\mathrm{P}<0.05)$; "\#" indicates there is no significant difference between the two groups $(\mathrm{P}>0.05)$. (F) Light microscopic images of nanoparticles before and after phase transformation caused by heating. 
528 Figure 4. The schematic diagram of the two-step pre-targeting technology.

529 (A) The schematic diagram of the two-step pre-targeting technology. (B) Confocal laser scanning 530 microscope images of human ovarian cancer SKOV3 cells showing the results of incubation with 531 FITC-labeled secondary antibodies. Green: FITC. Blue: DAPI.

532

533 Figure 5. The survival rate of SKOV3 cells after adding nanoparticles and targeting efficacy of 534 nanoparticles.

535 (A) Effects of different concentrations of PTX-PLGA/PFPs on the survival rate of SKOV3 cells 536 determined via CCK-8 assays. (B) The average cell fluorescence intensities detected with flow 537 cytometry. "*" indicates there is a significant difference compared with the other groups $538(\mathrm{P}<0.05)$; "\&" indicates there is a significant difference compared with these two groups $(\mathrm{P}<0.05)$. 539 Laser confocal microscope images of SKOV3 cells incubated with (C) Drug-loaded pretargeting, 540 (D) Drug-loaded and directly targeting, (E) Drug-loaded and non-targeting, and (F) Antibody 541 blocking.

Figure 6. Cancer cell survival rates determined with CCK-8 assays.

544 Cancer cell survival rates determined with CCK-8 assays. "*" indicates there is a significant 545 difference compared with the rest of the groups $(\mathrm{P}<0.05)$. 


\section{Figure 1}

Light microscope and electron microscope images of PTX-PLGA-SA/PFPs.

(A) light microscope image. (B) inverted fluorescence microscope image of PTX-PLGA-SA/PFPs labeled with Dil showing as red fluorescence. (C) SEM image. (D) TEM image. (E) Size distributions of PTXPLGA/PFPs, PLGA/PFPs, PTX-PLGA-Ab/PFPs, and PTX-PLGA-SA/PFPs. (F) The chromatography results for PTX encapsulated in nanoparticles in HPLC. (G) The release curve for PTX from PTXPLGA/PFPs in vitro. (H) Confocal laser scanning microscope images of PE labeled SA, Dil labeled PTX-PLGA-Ab/PFPs, Nanoparticles without fluorescence, and FITC labeled IgG. 
A

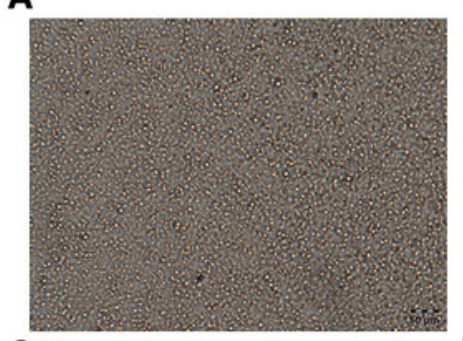

C

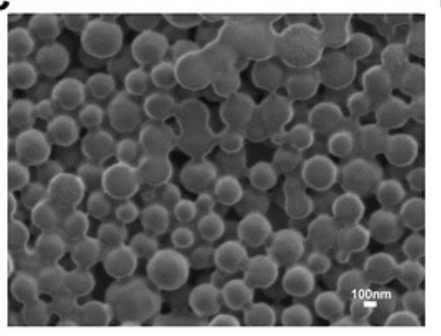

E
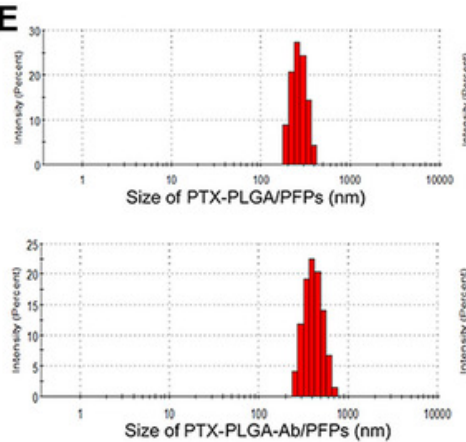

B

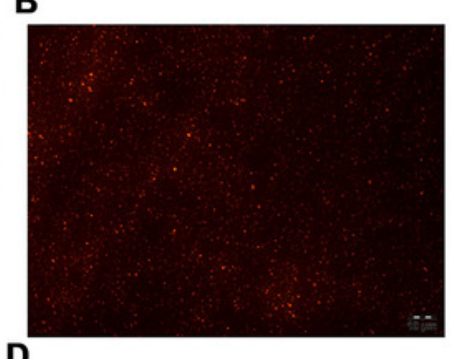

D
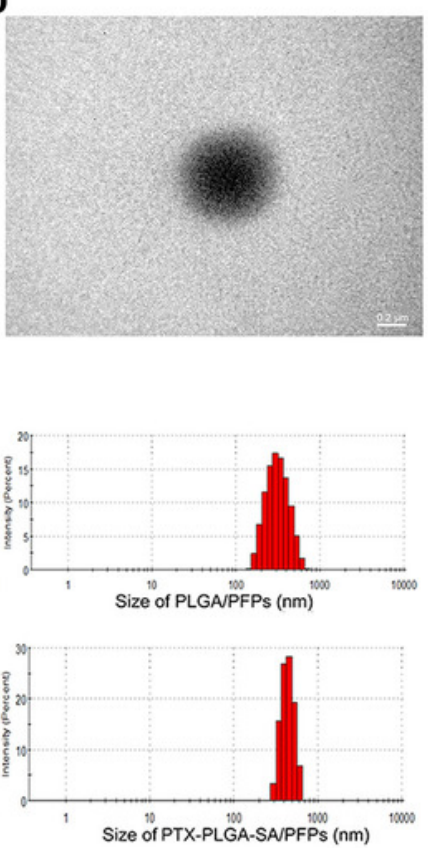

$\mathbf{F}$

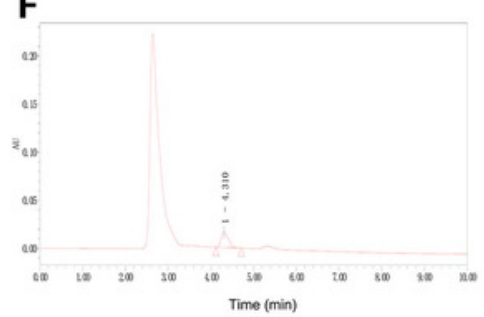

G

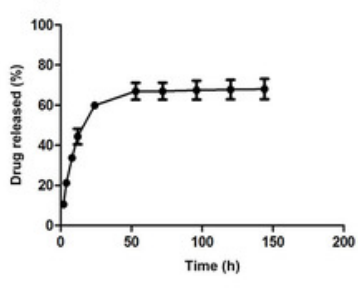

\section{H}

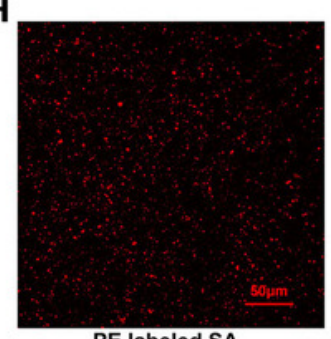

PE labeled SA

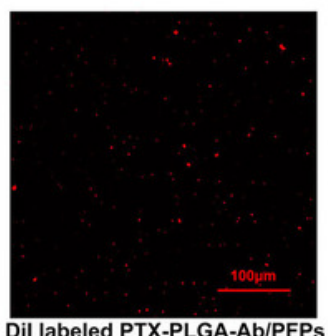

Dil labeled PTX-PLGA-Ab/PFPs
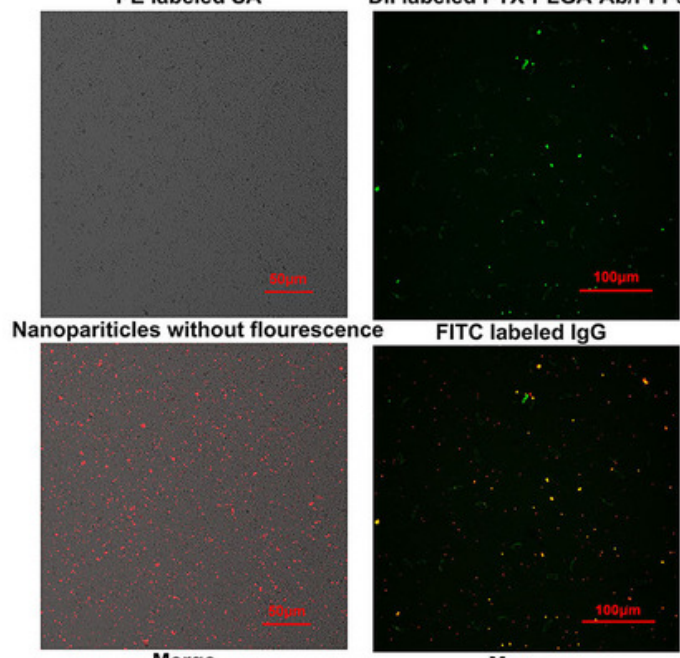

FITC labeled IgG

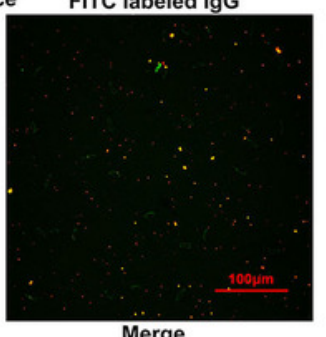


Figure 2

Flow cytometry analysis of the connection efficiency of both SA and Ab bound to PTXPLGA/PFPs.

(A) Control; (B) SA group; (C) Control; (D) Ab group. 

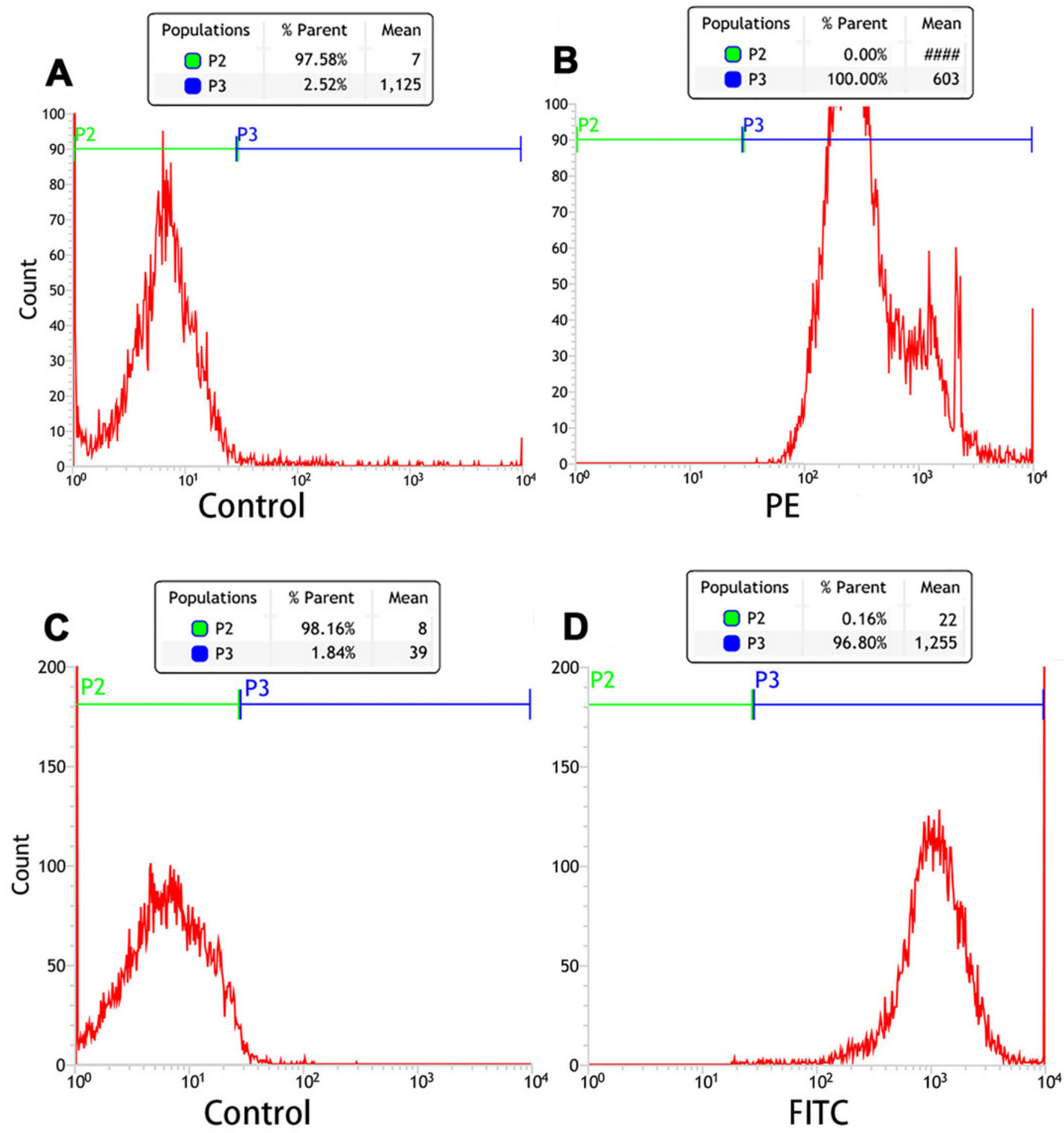


\section{Figure 3}

Phase shifting of PTX-PLGA-SA/PFPs.

$<!--[i f$ !supportLists]-->(A)<!--[endif]-->After exposure to LIFU at different power levels, the B-mode and contrast ultrasonic images of nanoparticles at 3.3, 4.4, 5.5, 6.5, 7.5, and $8.5 \mathrm{~W}$ are shown. After irradiation with LIFU at different power levels, the average echo intensities of the nanoparticles are shown. (B) B-mode; (C) CEUS mode. "** indicates there is a significant difference compared with the $0.1 \mathrm{mg} / \mathrm{ml}$ group $(\mathrm{P}<0.05)$; "\#" indicates there is no significant difference between the two groups $(P>0.05)$. " $\&$ " indicates there is a significant difference compared with the rest of the groups $(P<0.05)$. The average ultrasonic echo intensity $(\mathrm{dB})$ of the nanoparticles after phase transformation induced by LIFU at different concentrations. (D) B-mode; (E) CEUS. "*" indicates there is a significant difference compared with the $0.1 \mathrm{mg} / \mathrm{ml}$ group $(P<0.05)$; "\#" indicates there is no significant difference between the two groups ( $P>0.05)$. (F) Light microscopic images of nanoparticles before and after phase transformation caused by heating. 

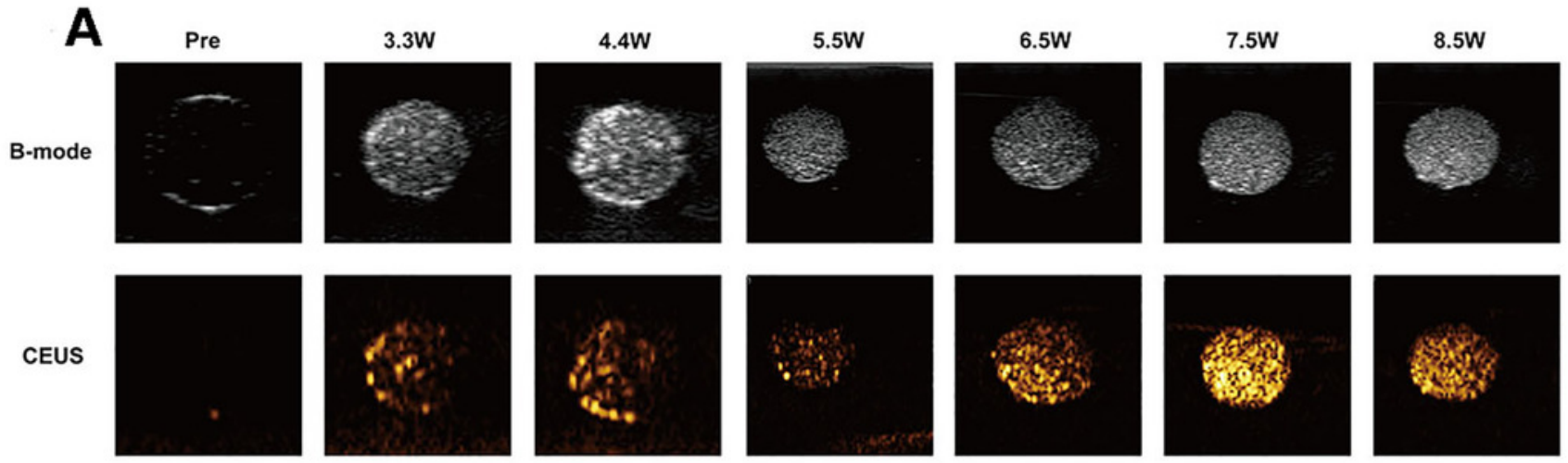

B

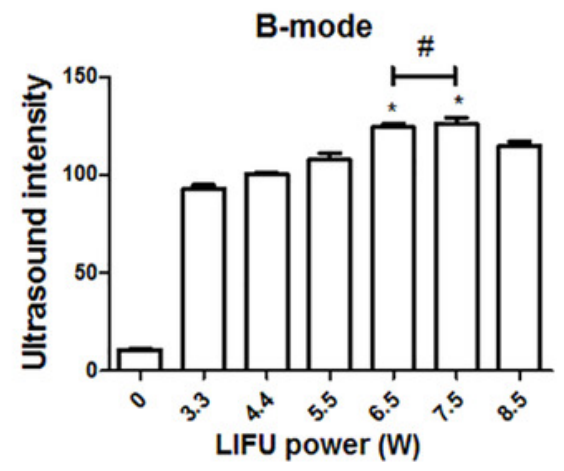

C

CEUS mode

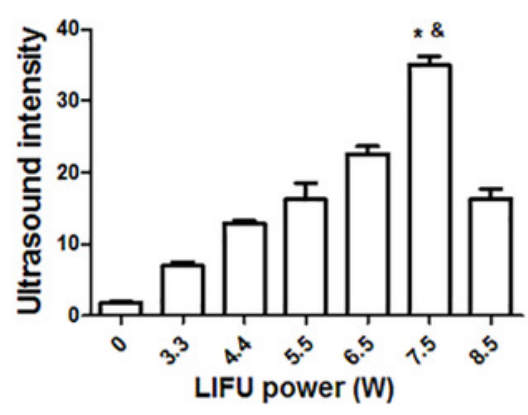

D

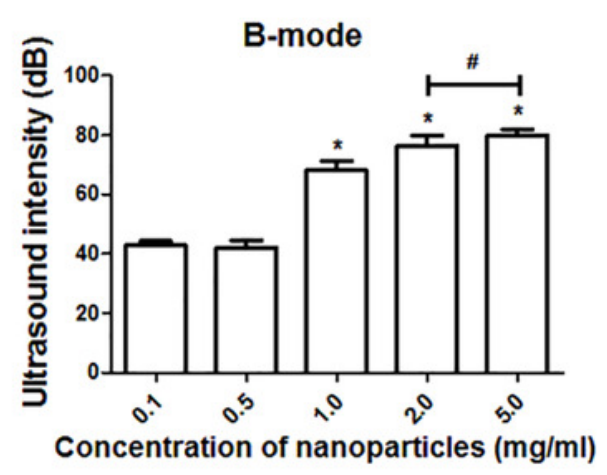

F
E

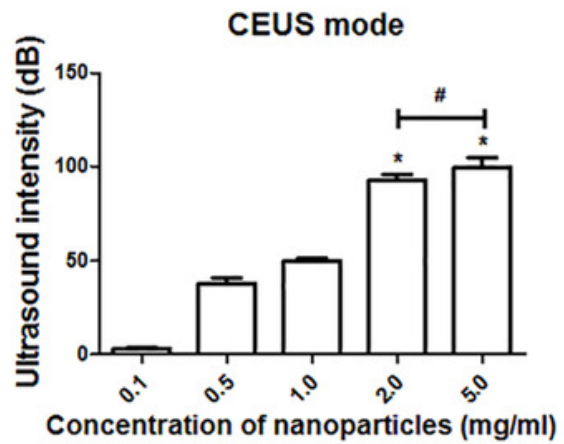

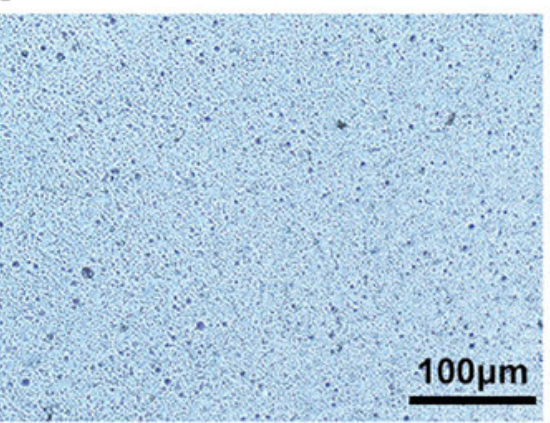
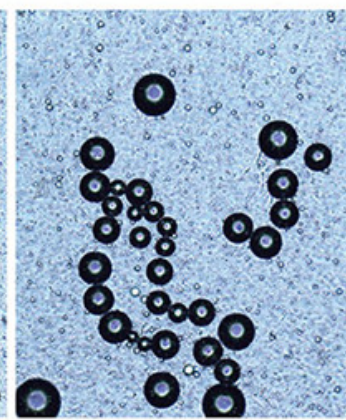

-

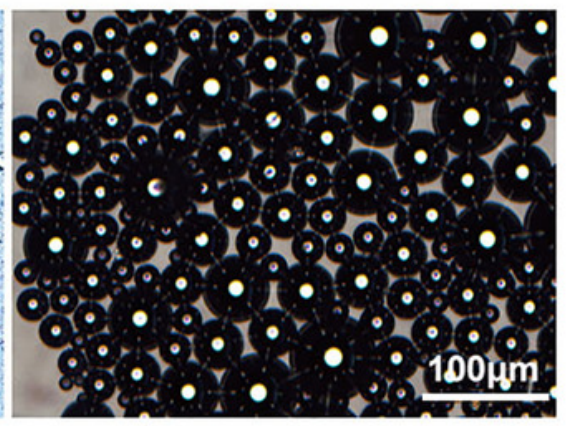


Figure 4

The schematic diagram of the two-step pre-targeting technology.

(A) The schematic diagram of the two-step pre-targeting technology. (B) Confocal laser

scanning microscope images of human ovarian cancer SKOV3 cells showing the results of incubation with FITC-labeled secondary antibodies. Green: FITC. Blue: DAPI. 
A

First Step: Bio-Abs pretarget to the CEA on the surface of the SKOV cells.
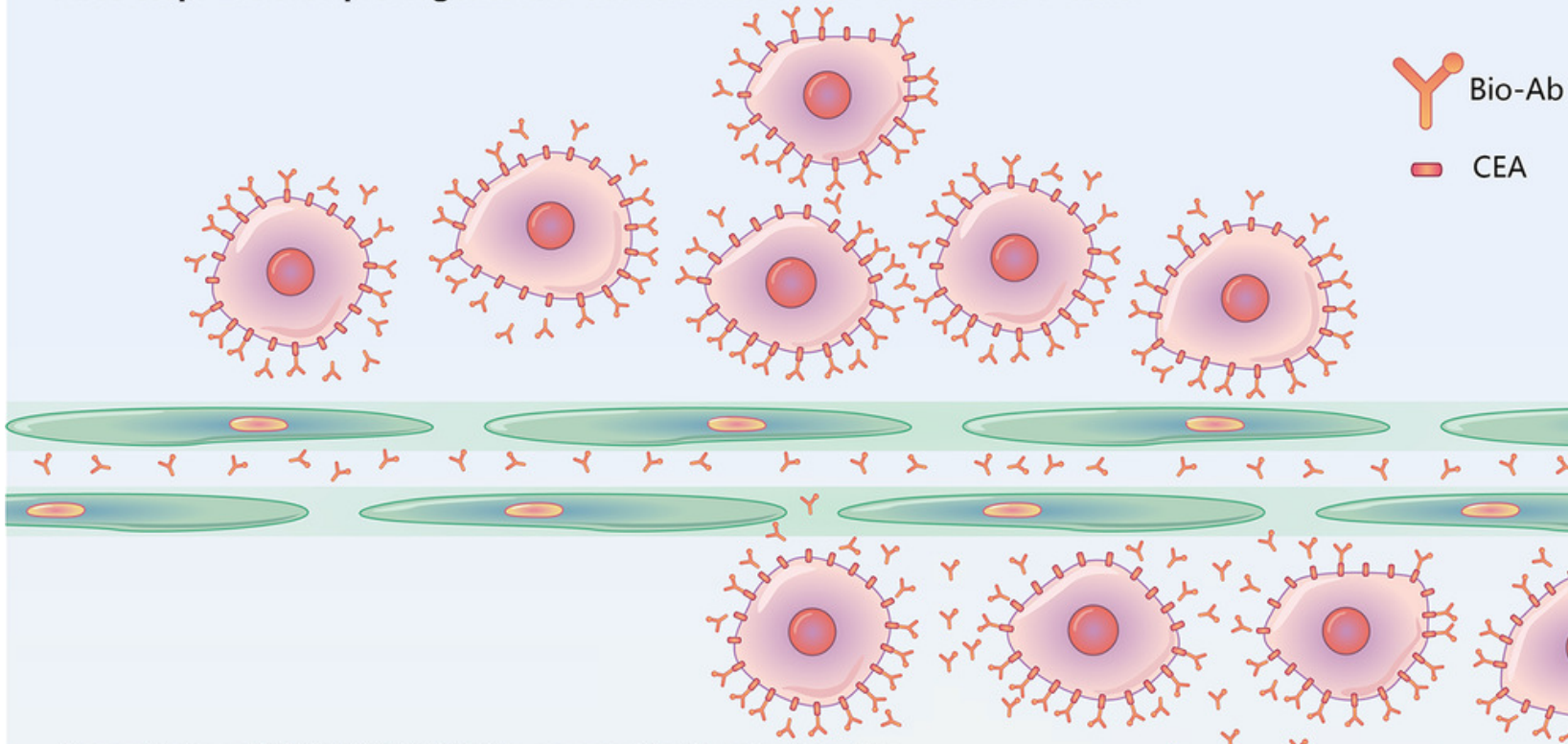

Second step: PTX-PLGA-SA/PFPs covalently bind to Bio-Abs.
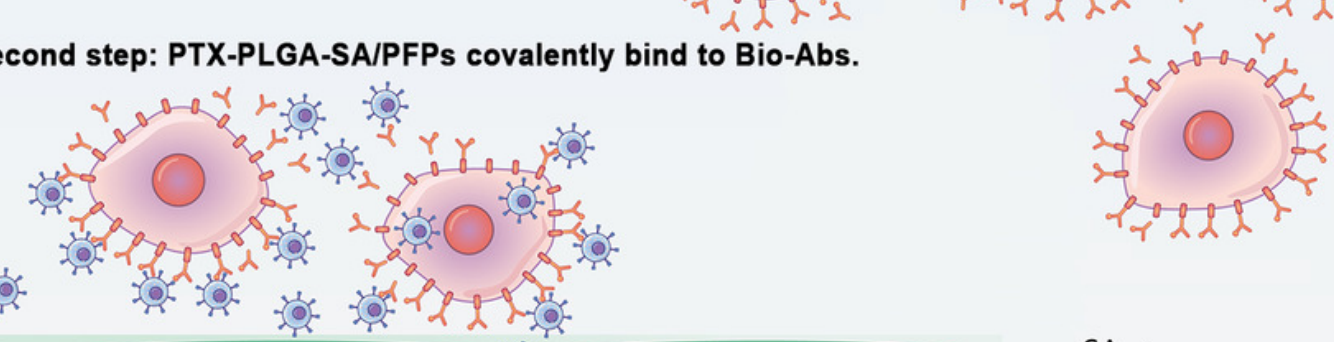

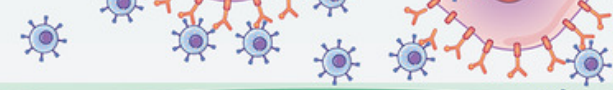
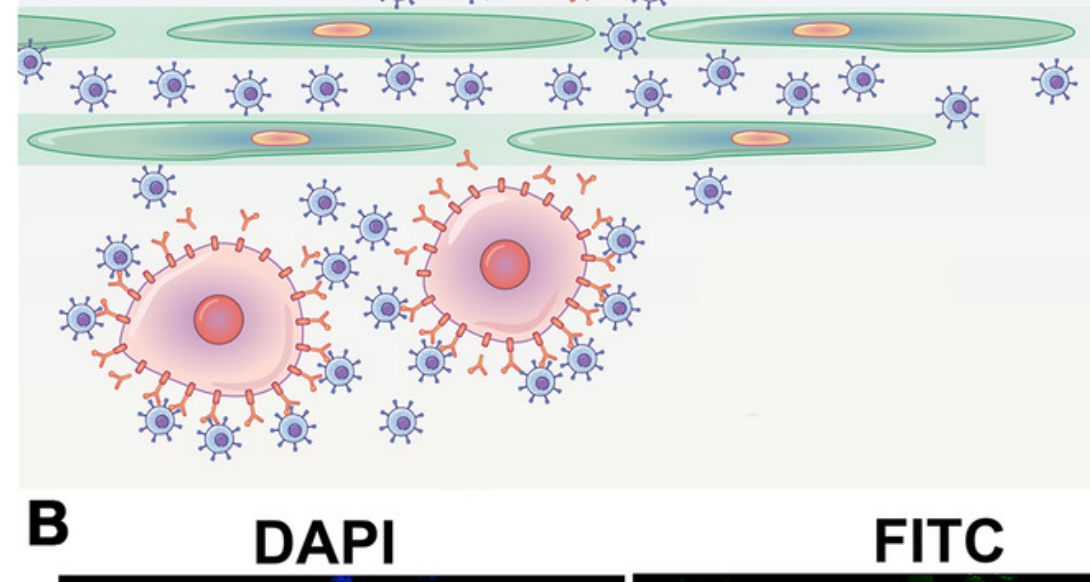

FITC

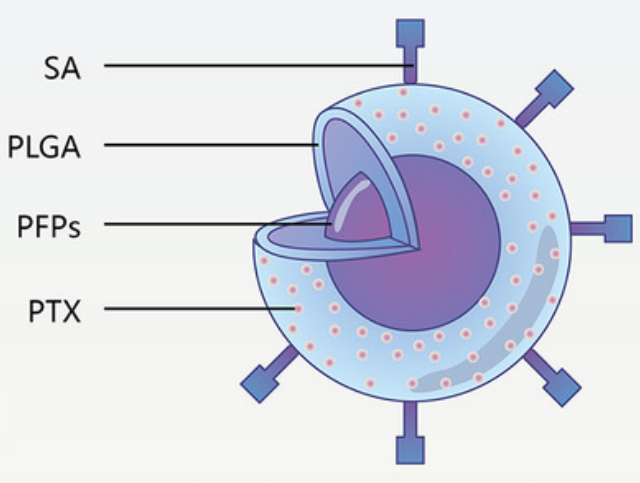

Stucture of PTX-PLGA-SA/PFPs
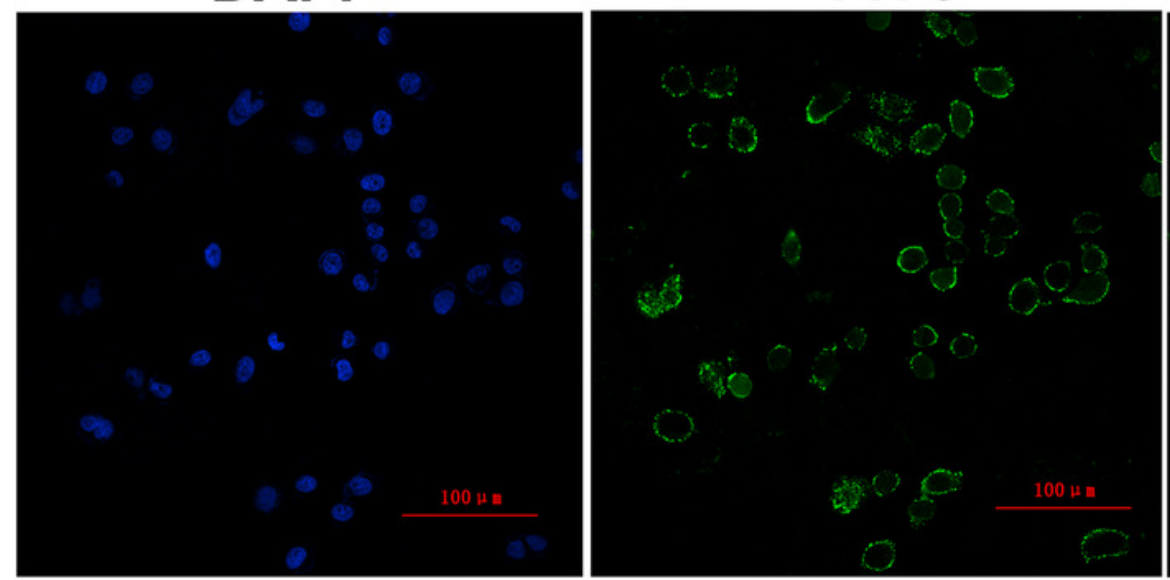

Merge

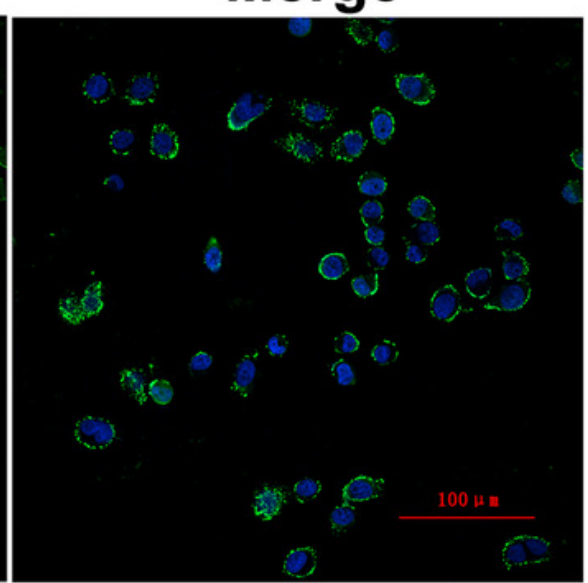




\section{Figure 5}

The survival rate of SKOV3 cells after adding nanoparticles and targeting efficacy of nanoparticles.

(A) Effects of different concentrations of PTX-PLGA/PFPs on the survival rate of SKOV3 cells determined via CCK-8 assays. (B) The average cell fluorescence intensities detected with flow cytometry. "*" indicates there is a significant difference compared with the other groups $(P<0.05)$; " $\&$ " indicates there is a significant difference compared with these two groups $(P<0.05)$. Laser confocal microscope images of SKOV3 cells incubated with $(C)$ Drug-loaded pretargeting, (D) Drug-loaded and directly targeting, (E) Drug-loaded and non-targeting, and (F) Antibody blocking. 


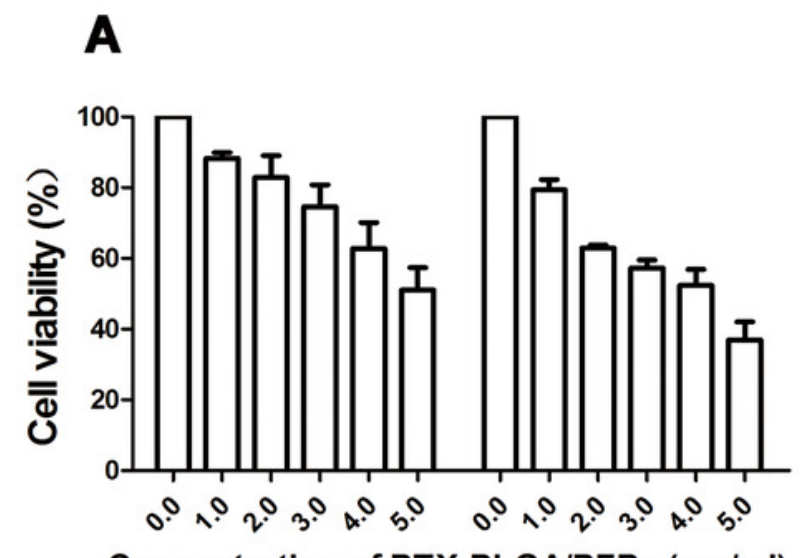

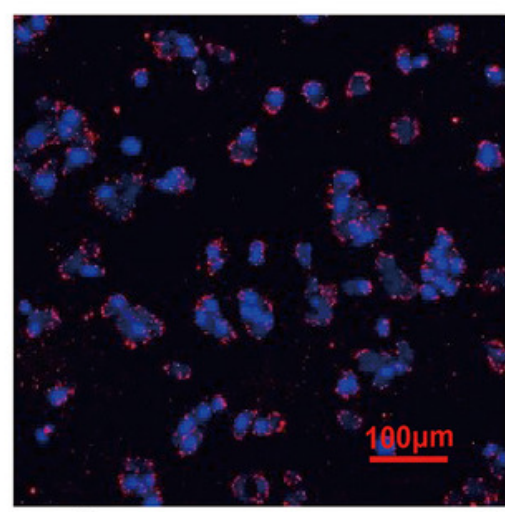

C Drug-loaded pretargeting

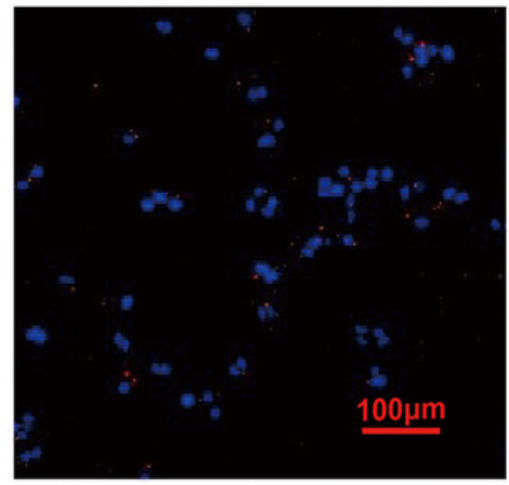

E Drug-loaded and non-targeting

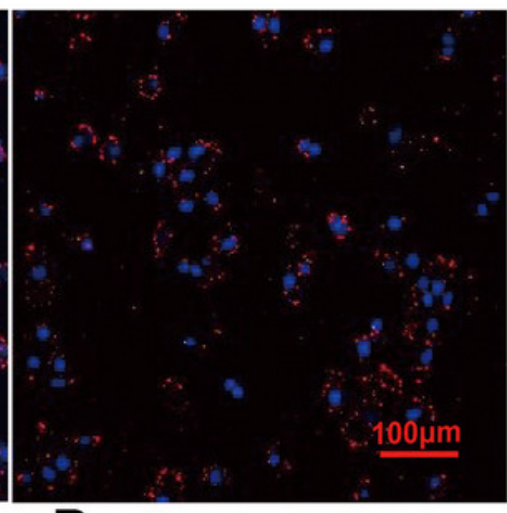

D Drug-loaded and directly targeting

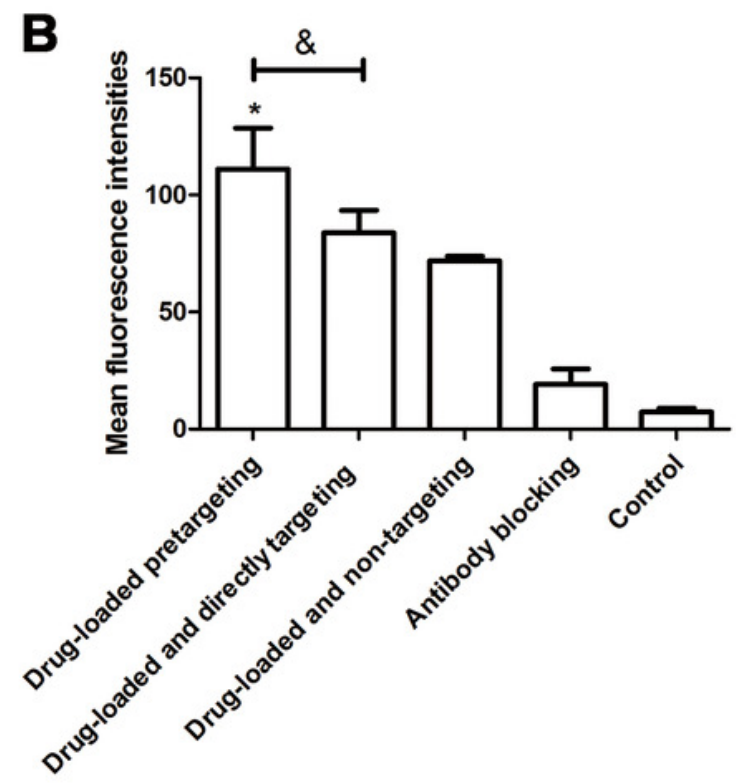

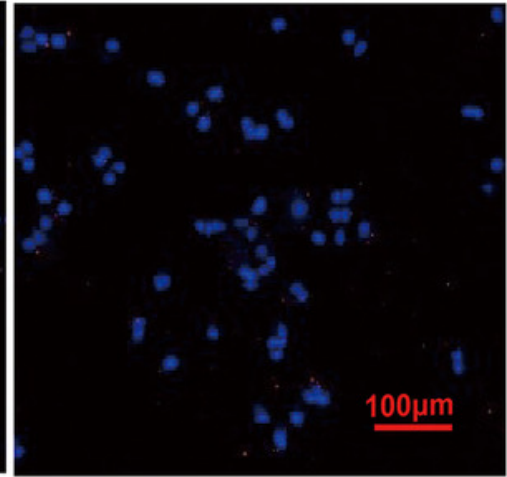

F Antibody blocking 
Figure 6

Cancer cell survival rates determined with CCK-8 assays.

Cancer cell survival rates determined with CCK-8 assays. "*" indicates there is a significant difference compared with the rest of the groups $(P<0.05)$.

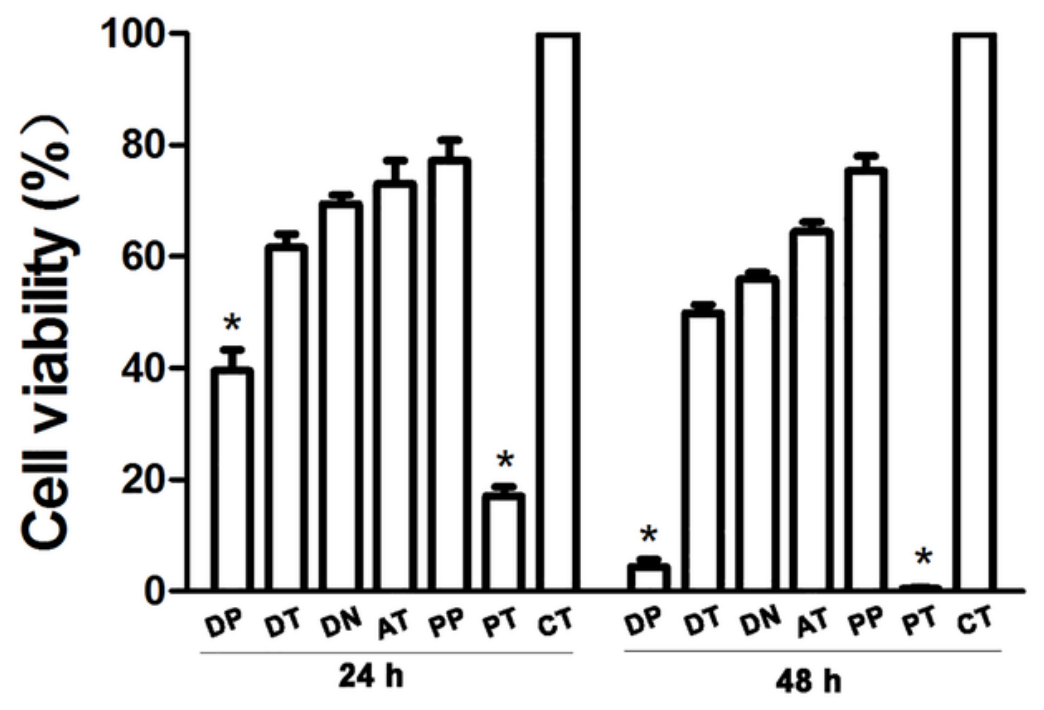

DP: Drug-loaded pretargeting DT: Drug-loaded and directly targeting DN: Drug-loaded and non-targeting AT: Antibody blocking PP: PLGAPFPs

PT: PTX CT: Control 